\title{
Linear and logarithmic entanglement production in an interacting chaotic system
}

\author{
Sanku Paul ${ }^{1}$ and Arnd Bäcker $\odot^{2,1}$ \\ ${ }^{1}$ Max-Planck-Institut für Physik komplexer Systeme, Nöthnitzer Straße 38, 01187 Dresden, Germany \\ ${ }^{2}$ Technische Universität Dresden, Institut für Theoretische Physik and Center for Dynamics, 01062 Dresden, Germany
}

(Received 29 July 2020; accepted 28 October 2020; published 24 November 2020)

\begin{abstract}
We investigate entanglement growth for a pair of coupled kicked rotors. For weak coupling, the growth of the entanglement entropy is found to be initially linear followed by a logarithmic growth. We calculate analytically the time after which the entanglement entropy changes its profile, and a good agreement with the numerical result is found. We further show that the different regimes of entanglement growth are associated with different rates of energy growth displayed by a rotor. At a large time, energy grows diffusively, which is preceded by an intermediate dynamical localization. The time span of intermediate dynamical localization decreases with increasing coupling strength. We argue that the observed diffusive energy growth is the result of one rotor acting as an environment to the other, which destroys the coherence. We show that the decay of the coherence is initially exponential followed by a power law.
\end{abstract}

DOI: 10.1103/PhysRevE.102.050102

Entanglement, as characterized by the von Neumann entropy, has recently emerged as an indispensable tool to distinguish phases and phase transitions in many-body quantum systems and reveals highly nonlocal information [1-3]. Many-body localization, which emerges due to ergodicity breaking, is known to exhibit a logarithmic growth of the entanglement entropy [4-9]. On the other hand, systems with thermalization show a saturation of entanglement growth [10]. Logarithmic slowdown is also observed in a system with longrange interaction along with a quench [11-13] and also in many-body system with nonergodic dynamics arising due to glassy behavior $[4,14]$.

On the other hand, systems with interactions, for which a finite speed of correlation spreading is generally observed $[9,11,15,16]$, show a linear growth of the entanglement entropy before saturating. Linear growth is also observed in open quantum systems [17-19], for instance, an inverted harmonic oscillator weakly coupled to a thermal bath [18]. It is further conjectured in Ref. [18] that the rate of linear growth equals the sum of positive Lyapunov exponents of the system. Similar correspondence between entropy production and the Lyapunov exponent has been shown for a kicked rotor coupled to a thermal bath [19]. However, coupled kicked tops show a violation of this conjecture, and it is observed that the rate depends on the coupling strength rather than on the Lyapunov exponents [20,21].

In addition to many-body and open quantum systems, even isolated two-body systems are capable of showing a non-

Published by the American Physical Society under the terms of the Creative Commons Attribution 4.0 International license. Further distribution of this work must maintain attribution to the author(s) and the published article's title, journal citation, and DOI. Open access publication funded by the Max Planck Society. trivial and often unexpected dynamics. For instance, coupled kicked rotors (CKR) have been found to display localizationdelocalization behavior depending on the coupling potential. For example, a CKR studied in Ref. [22] displays Anderson type of localization. A similar result is also seen in CKR with different coupling potential [23]. CKR with interaction in momentum space also show a localization with increased localization length compared to the single-particle localization length [24]. A point interaction in CKR results in dynamical localization of the center-of-mass momentum, which is destroyed for the relative momentum [25]. In contrast, there are systems displaying the destruction of localization resulting in momentum diffusion: For example, a CKR with a certain coupling potential shows a diffusive growth of the width of evolved state [26]. Similarly, for a spatially confined pair of $\delta$ kicked rotors, the center-of-mass motion displays destruction of localization [27]. Moreover, for CKR either localization or diffusion is reported, depending on the strength of coupling [28]. Experimentally realized CKR shows a localizationdelocalization transition [29]. Thus, the dynamics displayed by CKR depending on the coupling potential is not yet fully understood and in particular the relation to entanglement production as measure of quantum correlations between the two coupled particles has not been elucidated.

In this paper, we report on a surprising phenomenon in the entanglement production of a pair of coupled kicked rotors on a cylinder, which shows two distinct regimes of entanglement growth, i.e., linear and logarithmic, as time progresses. We show that this is tightly connected to a localization-delocalization crossover of time-evolved states with an intermediate dynamical localization. We also show that the logarithmic growth of the entanglement entropy commences once the system displays normal diffusion at large times, while before that, linear growth is found. We further show that, however weak the coupling is, the rotor will eventually display normal diffusion at large times. Analytically we 
calculate the growth of the linear entropy which shows an initial linear behavior followed by saturation. The rate of linear growth of the linear entropy is shown to depend quadratically on the ratio of scaled Planck's constant to the coupling strength rather than on the Lyapunov exponent. Furthermore, we provide an analytical expression for the time beyond which the logarithmic growth of the entanglement entropy starts.

A pair of coupled kicked rotors is a prototypical system for studying the dynamics and entanglement between two particles. Its Hamiltonian is given by

$$
\begin{aligned}
H= & \frac{p_{1}^{2}}{2}+\frac{p_{2}^{2}}{2}+\left[K_{1} \cos \left(x_{1}\right)+K_{2} \cos \left(x_{2}\right)\right. \\
& \left.+\xi_{12} \cos \left(x_{1}-x_{2}\right)\right] \sum_{n} \delta(t-n) \\
& =H_{1}+H_{2}+H_{12},
\end{aligned}
$$

where $H_{j}=\frac{p_{j}^{2}}{2}+K_{j} \cos \left(x_{j}\right) \sum_{n} \delta(t-n)$ represents the Hamiltonian of each kicked rotor and $H_{12}=\xi_{12} \cos \left(x_{1}-\right.$ $\left.x_{2}\right) \sum_{n} \delta(t-n)$ describes the coupling between the two rotors and it is in position space. The coupling can be interpreted as connecting the rotors by a spring. Here $p_{j}$ is the momentum and $x_{j}$ is the position of the $j$ th rotor. The kicking strengths of the kick received by $j$ th kicked rotor is $K_{j}$ and $\xi_{12}$ represents the coupling strength. By considering the dynamics stroboscopically, i.e., at multiple integer times, one obtains a four-dimensional symplectic map on a cylinder with periodic boundary conditions in the position coordinates. Note that by assuming in addition periodic boundary conditions in the momentum coordinates one obtains the four-dimensional coupled standard map [30-32].

For $\xi_{12}=0$, the system represents two uncoupled kicked rotors. If the kicking strengths $K_{j}$ of the individual rotors are sufficiently large, their dynamics is chaotic with a Lyapunov exponent of approximately $\ln \left(K_{j} / 2\right)$ [33]. In the following numerical investigations, we use $K_{1}=9.0$ and $K_{2}=10.0$ so that the classical dynamics is chaotic. In this chaotic case, the single or uncoupled kicked rotors display normal diffusion, i.e., a linear growth of the mean energy for an ensemble of initial conditions, $\langle E\rangle=D_{\mathrm{cl}} t[34,35]$, where $D_{\mathrm{cl}}$ is the classical diffusion coefficient.

For the quantum dynamics of CKR, the time evolution is given by the unitary operator $U=\left(U_{1} \otimes U_{2}\right) U_{12}$, where $U_{j}=$ $e^{-i \frac{p_{j}^{2}}{2 \hbar_{\mathrm{s}}}} e^{-i \frac{K_{j}}{\hbar_{\mathrm{s}}} \cos \left(x_{j}\right)}$ and $U_{12}=e^{-i \frac{\xi_{12}}{h_{\mathrm{s}}} \cos \left(x_{1}-x_{2}\right)}$, such that $|\Psi(t)\rangle=$ $U^{t}|\Psi(0)\rangle$ is the time-evolved state at discrete time $t$ of the initial state $|\Psi(0)\rangle$. In the following, we consider as initial state product states of the form $|\Psi(0)\rangle=\left|\psi_{1}(0)\right\rangle \otimes\left|\psi_{2}(0)\right\rangle$, where $\left|\psi_{j}(0)\right\rangle$ is a coherent state of the $j$ th rotor. For the numerical calculations, the fast Fourier transform is employed by expressing momentum and position values of each rotor evaluated on discrete grids of the same size $N=2^{l}$ with $l=11$. Note that in contrast to the normal diffusion displayed by a classical single kicked rotor, its quantum dynamics shows a suppression of the diffusion. This phenomenon is called dynamical localization, which is a phase-coherent effect analogous to Anderson localization observed in disordered lattices [36]. Also note that, similar to the classical case, the pair of quantum kicked rotors can also be considered with periodic boundary conditions in momentum $[32,37,38]$ and the spectral properties and entanglement generation in this case has been investigated in detail in Refs. [39-42].

The entanglement between the subsystems given by the two kicked rotors can be characterized by the von Neumann entropy

$$
S_{\mathrm{vN}}(t)=-\operatorname{Tr}_{1}\left(\rho_{1}(t) \log \rho_{1}(t)\right)
$$

where $\rho_{1}(t)=\operatorname{Tr}_{2}(\rho(t))$ is the reduced density matrix obtained by tracing out the contribution of second subsystem and $\rho(t)=|\Psi(t)\rangle\langle\Psi(t)|$ is the total density matrix of the time-evolved state $|\Psi(t)\rangle$.

Figure 1(a) shows that the growth of $S_{\mathrm{vN}}$ has two distinct regimes, linear and logarithmic. Initially, $S_{\mathrm{vN}}$ grows linearly up to a crossover time $t^{*}$; see the inset of Fig. 1(a) represented by a blue vertical dashed line. We find that the rate of this linear growth depends on the coupling strength following the relation $S_{\mathrm{vN}} \sim \xi_{12}^{\beta}$. The value of the exponent $\beta$ is numerically found to be approximately 1.85 . In this regime of linear growth, the rate turns out to be independent of the kicking strengths when both rotors display classically chaotic dynamics. After the break time $t^{*}$, the growth of $S_{\mathrm{vN}}$ slows down and shows a logarithmic dependence on time, i.e., $S_{\mathrm{vN}}=\frac{1}{2} \ln t+$ const. The value of $S_{\mathrm{vN}}$ at the onset of logarithmic growth, i.e., $S_{\mathrm{vN}}\left(t^{*}\right)$, depends on the quantum diffusion coefficient, which in turn depends on the kicking strength. Additionally, it is found that $S_{\mathrm{vN}}\left(t^{*}\right)$ barely depends on the coupling strength (not shown). Thus, the key finding is that the production of entanglement between the rotors does not follow a single functional form and, remarkably, in the two regimes a different dependency on the system parameters, i.e., $\xi_{12}$ and $K_{j}$, is found.

To obtain a qualitative understanding of the observed entanglement growth in terms of the underlying quantum dynamics, let us consider the behavior of mean energy growth $\left\langle E_{1}\right\rangle=\left\langle\Psi(t)\left|\frac{p_{1}^{2}}{2}\right| \Psi(t)\right\rangle$ and the distributions $g\left(x_{1}\right)=\left|\left\langle\Psi \mid x_{1}\right\rangle\right|^{2}$ in position space and $f\left(p_{1}\right)=\left|\left\langle\Psi \mid p_{1}\right\rangle\right|^{2}$ in momentum space of the first rotor. Figure 1(b) shows that there are three different regimes: Initially, $\left\langle E_{1}\right\rangle$ grows linearly until the break time $t_{\mathrm{b}}$, which is indicated by a orange vertical dash dotted line in Fig. 1(b). The break time is the time until which the quantum energy of a single kicked rotor follows the classical energy growth $[35,43]$. Until this time, the system builds up its quantum correlations, which after $t_{\mathrm{b}}$ leads to the emergence of an intermediate dynamical localization (IDL) for which $\left\langle E_{1}\right\rangle$ is essentially constant. This IDL extends up to the cross-over time $t^{*}$, indicated by a blue vertical dashed line in Fig. 1(b). Beyond $t^{*}$, the system displays normal diffusion, $\left\langle E_{1}\right\rangle \sim t$. Moreover, it can be seen from the inset of Fig. 1(b) that the temporal extent of the IDL increases with decreasing coupling $\xi_{12}$. An important consequence of this observation is that the system will always show normal diffusion at large times for any nonvanishing coupling $\xi_{12}>0$.

This result clearly shows that mapping CKR by performing two-dimensional (2d) Fourier transform onto a $2 \mathrm{~d}$ Anderson model does not necessarily imply localization. One reason is that in the present model, the coupling term in Eq. (1) modifies the hopping term of the corresponding $2 \mathrm{~d}$ Anderson model instead of the on-site energy term as in Ref. [24,44] or two 

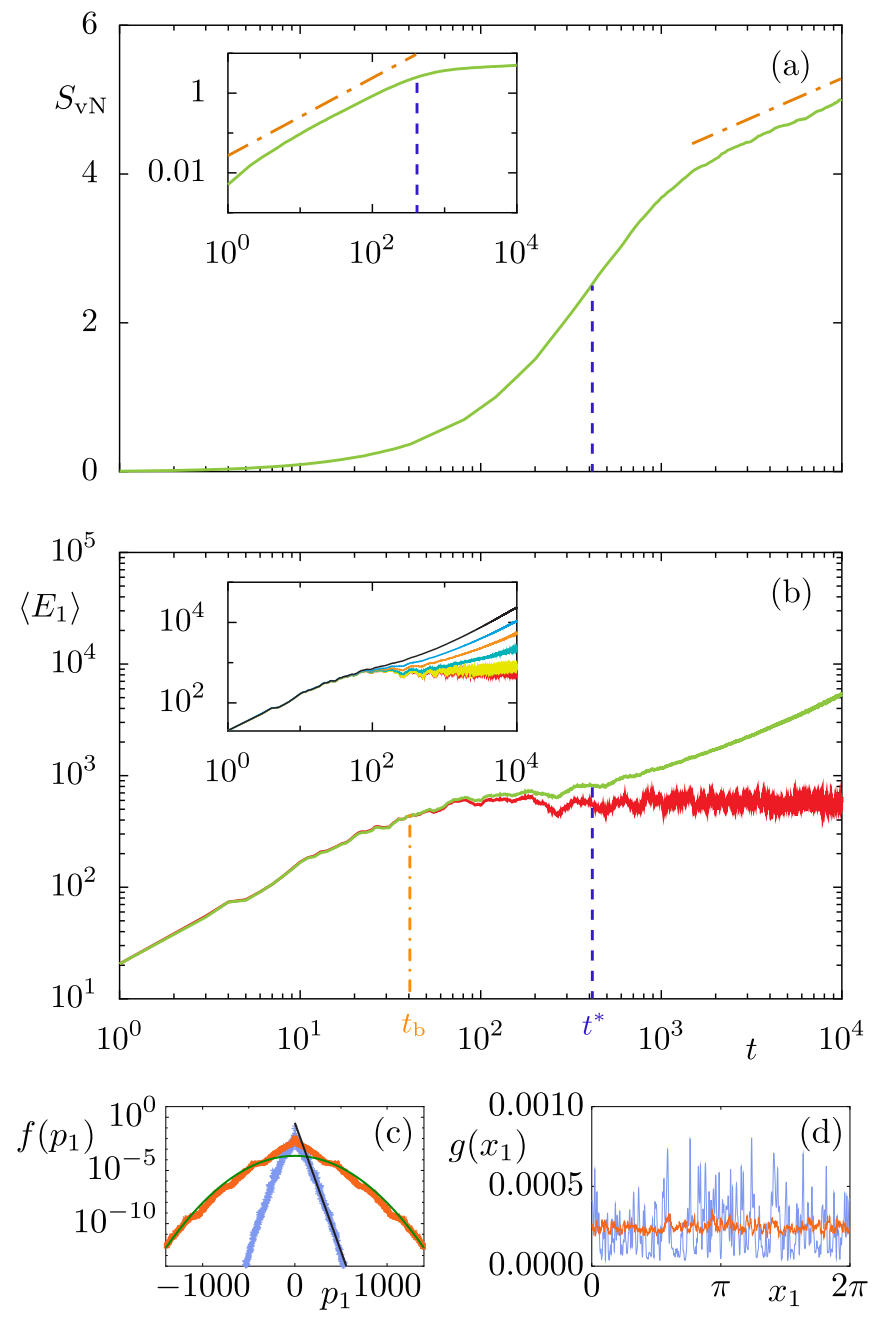

FIG. 1. (a) Entanglement entropy $S_{\mathrm{vN}}$ as function of time. The blue vertical dashed line represents the crossover time $t^{*}$ at which the transition from linear to logarithmic occurs. Inset captures the linear regime. The orange dash-dotted lines are linear fits to $S_{\mathrm{vN}}$. (b) Mean energy growth $\left\langle E_{1}\right\rangle$ of the first kicked rotor as a function of time. The orange dash-dotted line indicates the break time $t_{\mathrm{b}}$. Inset shows $\left\langle E_{1}\right\rangle$ for different coupling strengths, $\xi_{12}=$ $0.0,0.01,0.03,0.05,0.07,0.1$, from bottom to top. Panels (c) and (d) represent momentum and position distributions at two different times, $t=150$ (blue symbols) and $t=10000$ (orange symbols). The solid lines in panel (c) are Gaussian (green) and exponential (black) fits. All plots are for $K_{1}=9.0, K_{2}=10.0, \xi_{12}=0.05$ (for green solid line), and $\hbar_{\mathrm{s}}=1.0$.

particles in the 1d Anderson model [45]. In fact, in Ref. [46] it is shown that a nonlinearity in the off-diagonal terms in the 2D Anderson model leads to a subdiffusive wave-packet spreading, indicating that localization is not always guaranteed. In Ref. [22], the observed localization in CKR with coupling in position space is explained by mapping it onto a $2 \mathrm{~d}$ Anderson model. However, if one performs a coordinate transformation of the corresponding Hamiltonian to a center-of-mass frame, the coupling is in momentum space and the position space becomes decoupled. Thus, on mapping, it effectively modifies the on-site energy term instead of the hopping term. In contrast, for the system considered in this paper, even after coordinate transformation, it remains decoupled in momentum space and coupled in position space.

Another significant observation is that the normal diffusion seen in Fig. 1(b) is similar to classical diffusion. This can be seen from Fig. 1(c), which shows that the momentum distribution at time $t=10000$ is well described by a Gaussian. Also the position distribution becomes very uniform with only small quantum fluctuations; see Fig. 1(d). In contrast, the momentum distribution is exponentially localized in the IDL, as illustrated at $t=150$ in Fig. 1(c), and the corresponding position distribution shows much larger fluctuations, as seen in Fig. 1(d).

The Gaussian momentum and uniform position distributions are typical features of a corresponding classical diffusive regime [35]. Thus, the appearance of normal diffusion suggests that the rotors provide noise to each other, which destroys quantum coherence. Quantum coherence is the origin of the appearance of dynamical localization for a single kicked rotor. As a result, classical-like behavior emerges which in turn gives rise to a slow growth of $S_{\mathrm{vN}}$. Thus, we can conclude that the linear regime of $S_{\mathrm{vN}}$ appears when the system has quantum correlations. On the other hand, for $t>t^{*}$, where normal diffusion dominates, complete loss of correlations gives rise to the logarithmic growth.

Now, we provide a theoretical explanation of the emergence of the two regimes of $S_{\mathrm{vN}}$ growth in the case of weak coupling $\xi_{12} \ll 1$. For this, we consider the linear entropy $S_{\text {lin }}(t)=\operatorname{Tr}_{1} \rho_{1}(t)^{2}$, which is analytically easier tractable than the von Neumann entropy $S_{\mathrm{vN}}$, but shows the same characteristics. To treat the initial time dependence, the key point is to consider that one rotor acts as an environment to the other, so that we can rewrite the Hamiltonian in Eq. (1) as $H=H_{\mathrm{S}}+H_{\mathrm{E}}+V(t)$, where $H_{\mathrm{S}}\left(H_{\mathrm{E}}\right)$ represents the system (environment) Hamiltonian and $V(t)$ is the interaction. The evolution of the total density matrix $\rho(t)$ in the interaction picture is

$$
\frac{d \rho(t)}{d t}=-\frac{i}{\hbar_{\mathrm{s}}}[V(t), \rho(t)] .
$$

As the initial state is a product state, we have $\rho(0)=\rho_{\mathrm{S}}(0) \otimes$ $\rho_{\mathrm{E}}(0)$. Performing formal integration and iteration and considering $\xi_{12} \ll 1$, we arrive at

$$
\begin{aligned}
\rho(t)= & \rho(0)-\frac{i \xi_{12}}{\hbar_{\mathrm{s}}} \sum_{r=1}^{t}[\mathcal{F}(r), \rho(0)] \\
& +\left(\frac{i \xi_{12}}{\hbar_{\mathrm{s}}}\right)^{2} \sum_{r=1}^{t} \sum_{s=1}^{r-1}[\mathcal{F}(s),[\mathcal{F}(r), \rho(0)]],
\end{aligned}
$$

where $\mathcal{F}(r)=\cos \left(x_{1}(r)-x_{2}(r)\right)$. The summation instead of integration that appears in Eq. (4) is due to the fact that coupling acts only at integer times, i.e., $V(t)=\xi_{12} \cos \left(x_{1}-\right.$ $\left.x_{2}\right) \sum_{n} \delta(t-n)$. The calculation of $\rho(t)$ is most conveniently done in the position basis as the interaction is in position space. With $\rho_{\mathrm{S}}(t)=\operatorname{Tr}_{E} \rho(t)$, the computation of $S_{\text {lin }}=1-$ $\operatorname{Tr}_{\mathrm{S}}\left(\rho_{\mathrm{S}}(t)^{2}\right)$ leads to

$$
S_{\operatorname{lin}}(t)=\left(\frac{\xi_{12}}{\hbar_{\mathrm{s}}}\right)^{2} C(t),
$$


where $C(t)=\sum_{r, s=1}^{t} C(r, s)$ and $C(r, s)$ represents the correlation function at two different time steps. If both rotors display classically chaotic dynamics, then $C(t)$ is independent of the system parameters. Furthermore, it is numerically found that for small coupling, $C(t)$ depends linearly on time. Thus, Eq. (5) reveals that the rate $\Gamma=\frac{d S_{\text {lin }}}{d t}$ depends only on the ratio $\frac{\xi_{12}}{\hbar_{\mathrm{s}}}$ rather than on the kicking strengths $K_{j}$. This implies that for weak coupling the initial temporal growth of $S_{\text {lin }}$ does not depend on the strength of chaos of the individual rotors.

To determine the behavior of the linear entropy at large times, i.e., for $t>t^{*}$, we employ that the time-evolved initial state becomes on average Gaussian in momentum and uniform in position space; see Fig. 1(c) and 1(d). Using the Husimi function $\mathcal{H}\left(x_{1}, p_{1}\right)$ one can express the linear entropy as [47]

$$
S_{\text {lin }}=1-\int \mathcal{H}\left(x_{1}, p_{1}\right)^{2} \frac{d p_{1} d x_{1}}{2 \pi \hbar_{\mathrm{s}}} .
$$

We approximate the Husimi distribution of the time-evolved state by $\mathcal{H}\left(x_{1}, p_{1}\right)=\frac{\hbar_{\mathrm{s}}}{\sqrt{2 \pi D_{\mathrm{q}} t}} \exp \left(-\frac{p_{1}^{2}}{2 D_{\mathrm{q}} t}\right)$, where $D_{\mathrm{q}}$ is the quantum diffusion coefficient. Inserting $\mathcal{H}\left(x_{1}, p_{1}\right)$ in Eq. (6) gives

$$
S_{\text {lin }}=1-\frac{\hbar_{\mathrm{s}}}{\sqrt{4 \pi D_{\mathrm{q}}}} t^{-1 / 2} .
$$

Equation (7) reveals that $S_{\text {lin }}$ in the regime of linear growth of $S_{\mathrm{vN}}$ depends on $D_{\mathrm{q}}$ and shows that $S_{\text {lin }}$ saturates at large times.

Equating the two expressions for $S_{\text {lin }}$ obtained in Eqs. (5) and (7) at $t=t^{*}$, at which the crossover occurs, and solving for $t^{*}$, we obtain

$$
t^{*}=\frac{\hbar_{\mathrm{s}}^{2}}{3 \xi_{12}^{2}}\left[2+\frac{1}{\mathcal{G}\left(\xi_{12}, D_{\mathrm{q}}\right)}+\mathcal{G}\left(\xi_{12}, D_{\mathrm{q}}\right)\right]
$$

where

$$
\mathcal{G}\left(\xi_{12}, D_{\mathrm{q}}\right)=\left[-1+\frac{27}{8 \pi} \frac{\xi_{12}^{2}}{D_{\mathrm{q}}}+\frac{3^{\frac{3}{2}} \xi_{12}}{2} \sqrt{\frac{-1}{\pi D_{\mathrm{q}}}+\frac{27}{16 \pi^{2}} \frac{\xi_{12}^{2}}{D_{\mathrm{q}}^{2}}}\right]^{\frac{1}{3}} .
$$

Hence, $t^{*}$ depends on the coupling strength $\xi_{12}$, the scaled Planck's constant $\hbar_{\mathrm{s}}$, and the quantum diffusion coefficient $D_{\mathrm{q}}$. Equation (8) shows that with decreasing $\hbar_{\mathrm{s}}$ the value of $t^{*}$ decreases. This is because quantum correlations vanish in the semiclassical limit. Also, with increasing $\xi_{12}$, the crossover time $t^{*}$ decreases. This relates to the fact that the coupling between the subsystems destroys the coherence in the system. Figure 2 compares the analytical result (8) with numerical result and a very good agreement is found. This shows that, however small the coupling is, the system will eventually show normal diffusion. It is also interesting to note that for a noisy kicked rotor with noise strength $\epsilon$, the diffusion commences at a timescale $\frac{\hbar_{\mathrm{s}}^{2}}{\epsilon^{2}}$ [48].

As discussed, coherence plays a central role in the emergence of the different regimes of the entanglement growth as characterized by $S_{\mathrm{vN}}$ or $S_{\text {lin }}$. To investigate the decay of coherence and examine the nature of noise provided by one rotor to the other, we study the decay of the off-diagonal elements of $\rho_{1}$. The off-diagonal elements represent the interference between the system and the environment, and their decay indicates the loss of coherence [49]. We quantify this deco-

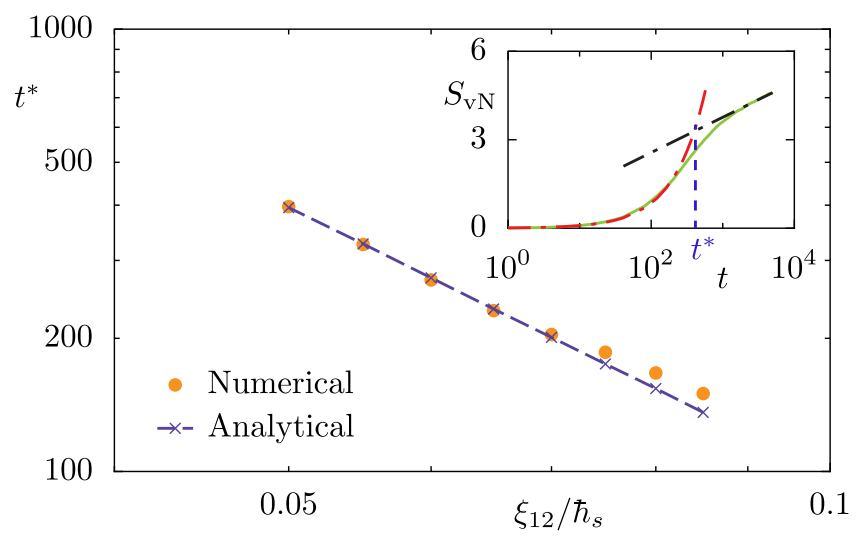

FIG. 2. Dependence of the cross-over time $t^{*}$ on the ratio of the coupling strength $\xi_{12}$ to the scaled Planck's constant $\hbar_{\mathrm{s}}$. The analytical result (blue dashed line with crosses) is compared to the numerical results (orange circles). The inset sketches the procedure to calculate $t^{*}$ numerically. Parameters are $K_{1}=9.0, K_{2}=10.0$, and $\hbar_{\mathrm{s}}=1.0$.

herence by calculating $\mathcal{D}(t)=\sum_{i \neq j} \rho_{1}^{i j}(t)$, which is shown in Fig. 3. Initially $\mathcal{D}(t)$ is close to one and follows an exponential decay until $t<t^{*}$. The exponential decay of coherence is also observed in a kicked rotor system with random noise [50,51]. This suggests that one rotor provides random noise to the other and thus effectively acts as an environment. Now, around $t=t^{*}$ in Fig. 3, one observes an extended transition and finally a power-law decay. The exponent of the power law is numerically found to be approximately 0.5 . This slow decay of coherence implies that the quantum system enters the classical-like regime as illustrated in Fig. 1(b) and will require an arbitrarily large time to actually behave like a classical system.

A closer look at the initial time dependence of the decoherence, as shown in the inset of Fig. 3, reveals an initial production of coherence for uncoupled rotors (red curve) until the break time $t_{\mathrm{b}}$ and then saturation to a constant value.

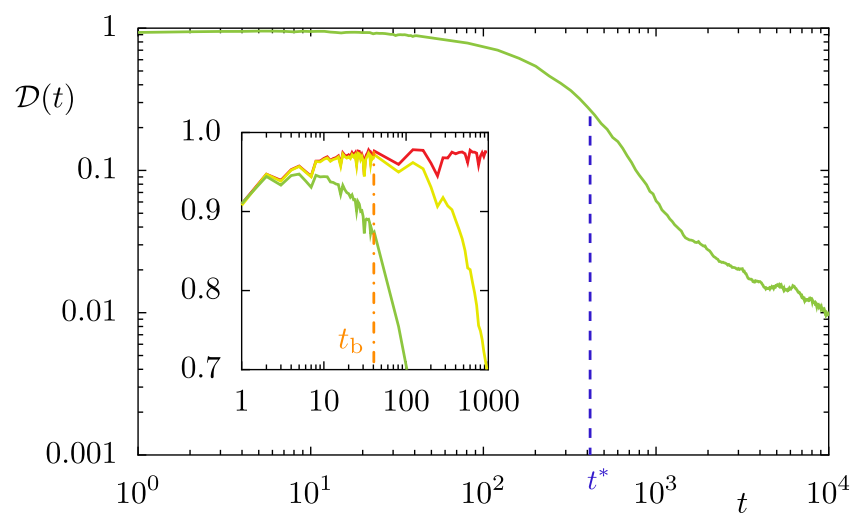

FIG. 3. Decay of coherence of the first kicked rotor for $\xi_{12}=$ 0.05 . The blue vertical dashed line represents $t^{*}$. The red, yellow, and green curves in the inset show the decoherence for $\xi_{12}=$ $0.0,0.01,0.05$, respectively. The orange vertical dash dotted line indicates the break time $t_{\mathrm{b}}$. Parameters are $K_{1}=9.0, K_{2}=10.0$, and $\hbar_{\mathrm{s}}=1.0$. 
This initial increase can also be observed in a weak coupling situation (yellow curve) for which $t_{\mathrm{b}} \ll t^{*}$ and because of that, a signature of IDL is observed as in Fig. 1(b) for $t>t_{\mathrm{b}}$. Even for coupling $\xi_{12}=0.05$, an initial increase in coherence for a small time interval $t<t_{\mathrm{b}}$ can be observed (green curve in the inset of Fig. 3) which corresponds to the appearance of a short IDL in Fig. 1(b). However, for strong coupling, the cross-over time $t^{*}$ becomes so small that an initial production of coherence is not possible. Thus, the coherence decays from the very beginning.

To summarize, for a pair of coupled kicked rotors, we demonstrate that the entanglement entropy shows two distinct regimes, initially linear growth followed by a logarithmic increase. The logarithmic regime sets in when the time-evolved state shows a Gaussian profile in momentum space and is uniform on average in position space. This regime can be considered as a kind of classical behavior caused by one rotor acting as a noisy environment to the other. This leads to exponential decoherence, which is clearly confirmed by the numerical results. The crossover time $t^{*}$ between linear and logarithmic behavior of the von Neumann entropy is computed using the linear entropy. Explicit expressions for $S_{\text {lin }}$ in both regimes are obtained and excellent agreement of the prediction for $t^{*}$ with numerics is found. Thus, we show that entanglement entropy allows us to distinguish two completely different dynamics, quantum and classical-like. It would be very interesting to experimentally investigate this CKR, for example, using ultracold atoms.

We thank Roland Ketzmerick, Arul Lakshminarayan, and David Luitz for useful discussions.
[1] R. Horodecki, P. Horodecki, M. Horodecki, and K. Horodecki, Quantum entanglement, Rev. Mod. Phys. 81, 865 (2009).

[2] N. Laflorencie, Quantum entanglement in condensed matter systems, Phys. Rep. 646, 1 (2016).

[3] Z.-C. Yang, C. Chamon, A. Hamma, and E. R. Mucciolo, TwoComponent Structure in the Entanglement Spectrum of Highly Excited States, Phys. Rev. Lett. 115, 267206 (2015).

[4] J. H. Bardarson, F. Pollmann, and J. E. Moore, Unbounded Growth of Entanglement in Models of Many-Body Localization, Phys. Rev. Lett. 109, 017202 (2012).

[5] M. Serbyn, Z. Papić, and D. A. Abanin, Universal Slow Growth of Entanglement in Interacting Strongly Disordered Systems, Phys. Rev. Lett. 110, 260601 (2013).

[6] R. Vosk and E. Altman, Many-Body Localization in One Dimension as a Dynamical Renormalization Group Fixed Point, Phys. Rev. Lett. 110, 067204 (2013).

[7] M. Žnidarič, T. Prosen, and P. Prelovšek, Many-body localization in the Heisenberg $X X Z$ magnet in a random field, Phys. Rev. B 77, 064426 (2008).

[8] A. Lukin, M. Rispoli, R. Schittko, M. E. Tai, A. M. Kaufman, S. Choi, V. Khemani, J. Léonard, and M. Greiner, Probing entanglement in a many-body-localized system, Science 364, 256 (2019).

[9] D. J. Luitz, N. Laflorencie, and F. Alet, Extended slow dynamical regime close to the many-body localization transition, Phys. Rev. B 93, 060201(R) (2016).

[10] R. Singh, J. H. Bardarson, and F. Pollmann, Signatures of the many-body localization transition in the dynamics of entanglement and bipartite fluctuations, New J. Phys. 18, 023046 (2016).

[11] J. Schachenmayer, B. P. Lanyon, C. F. Roos, and A. J. Daley, Entanglement Growth in Quench Dynamics with Variable Range Interactions, Phys. Rev. X 3, 031015 (2013).

[12] A. Lerose and S. Pappalardi, Origin of the slow growth of entanglement entropy in long-range interacting spin systems, Phys. Rev. Res. 2, 012041 (2020).

[13] A. Lerose, J. Marino, A. Gambassi, and A. Silva, Prethermal quantum many-body Kapitza phases of periodically driven spin systems, Phys. Rev. B 100, 104306 (2019).

[14] M. van Horssen, E. Levi, and J. P. Garrahan, Dynamics of many-body localization in a translation-invariant quantum glass model, Phys. Rev. B 92, 100305(R) (2015).
[15] A. Nahum, J. Ruhman, S. Vijay, and J. Haah, Quantum Entanglement Growth under Random Unitary Dynamics, Phys. Rev. X 7, 031016 (2017).

[16] P. Calabrese and J. Cardy, Evolution of entanglement entropy in one-dimensional systems, J. Stat. Mech. (2005) P04010.

[17] E. Bianchi, L. Hackl, and N. Yokomizo, Linear growth of the entanglement entropy and the Kolmogorov-Sinai rate, J. High Energy Phys. 03 (2018) 025.

[18] W. H. Zurek and J. P. Paz, Decoherence, Chaos, and the Second Law, Phys. Rev. Lett. 72, 2508 (1994).

[19] P. A. Miller and S. Sarkar, Entropy production, dynamical localization, and criteria for quantum chaos in the open quantum kicked rotor, Nonlinearity 12, 419 (1999).

[20] H. Fujisaki, A. Tanaka, and T. Miyadera, Dynamical aspects of quantum entanglement for coupled mapping systems, J. Phys. Soc. Jpn. 72, 111 (2003).

[21] H. Fujisaki, T. Miyadera, and A. Tanaka, Dynamical aspects of quantum entanglement for weakly coupled kicked tops, Phys. Rev. E 67, 066201 (2003).

[22] E. Doron and S. Fishman, Anderson Localization for a TwoDimensional Rotor, Phys. Rev. Lett. 60, 867 (1988).

[23] S. Notarnicola, F. Iemini, D. Rossini, R. Fazio, A. Silva, and A. Russomanno, From localization to anomalous diffusion in the dynamics of coupled kicked rotors, Phys. Rev. E 97, 022202 (2018).

[24] F. Borgonovi and D. L. Shepelyansky, Enhancement of localization length for two interacting kicked rotators, Nonlinearity 8, 877 (1995).

[25] P. Qin, A. Andreanov, H. C. Park, and S. Flach, Interacting ultracold atomic kicked rotors: Loss of dynamical localization, Sci. Rep. 7, 41139 (2017).

[26] S. Adachi, M. Toda, and K. Ikeda, Quantum-Classical Correspondence in Many-Dimensional Quantum Chaos, Phys. Rev. Lett. 61, 659 (1988).

[27] H.-K. Park and S. W. Kim, Decoherence from chaotic internal dynamics in two coupled $\delta$-function-kicked rotors, Phys. Rev. A 67, 060102(R) (2003).

[28] B. Toloui and L. E. Ballentine, Quantum localization for two coupled kicked rotors, arXiv:0903.4632 [quant-ph].

[29] B. Gadway, J. Reeves, L. Krinner, and D. Schneble, Evidence for a Quantum-To-Classical Transition in a Pair of 
Coupled Quantum Rotors, Phys. Rev. Lett. 110, 190401 (2013).

[30] C. Froeschle, On the number of isolating integrals in systems with three degrees of freedom, Astrophys. Space Sci. 14, 110 (1971).

[31] C. Froeschlé, Numerical study of a four-dimensional mapping, Astron. Astrophys. 16, 172 (1972).

[32] M. Richter, S. Lange, A. Bäcker, and R. Ketzmerick, Visualization and comparison of classical structures and quantum states of four-dimensional maps, Phys. Rev. E 89, 022902 (2014).

[33] B. V. Chirikov, A universal instability of many-dimensional oscillator systems, Phys. Rep. 52, 263 (1979).

[34] G. Casati, B. Chirikov, F. Izraelev, and J. Ford, Stochastic behavior of a quantum pendulum under a periodic perturbation, in Stochastic Behavior in Classical and Quantum Hamiltonian Systems, edited by G. Casati and J. Ford, Lecture Notes in Physic Vol. 93 (Springer, Berlin, 1979), p. 334.

[35] F. M. Izrailev, Simple models of quantum chaos: Spectrum and eigenfunctions, Phys. Rep. 196, 299 (1990).

[36] D. R. Grempel, R. E. Prange, and S. Fishman, Quantum dynamics of a nonintegrable system, Phys. Rev. A 29, 1639 (1984).

[37] S.-J. Chang and K.-J. Shi, Evolution and exact eigenstates of a resonant quantum system, Phys. Rev. A 34, 7 (1986).

[38] A. Lakshminarayan, Entangling power of quantized chaotic systems, Phys. Rev. E 64, 036207 (2001).

[39] S. C. L. Srivastava, S. Tomsovic, A. Lakshminarayan, R. Ketzmerick, and A. Bäcker, Universal Scaling of Spectral Fluctuation Transitions for Interacting Chaotic Systems, Phys. Rev. Lett. 116, 054101 (2016).

[40] A. Lakshminarayan, S. C. L. Srivastava, R. Ketzmerick, A. Bäcker, and S. Tomsovic, Entanglement and localization transitions in eigenstates of interacting chaotic systems, Phys. Rev. E 94, 010205(R) (2016).

[41] S. Tomsovic, A. Lakshminarayan, S. C. L. Srivastava, and A. Bäcker, Eigenstate entanglement between quantum chaotic subsystems: Universal transitions and power laws in the entanglement spectrum, Phys. Rev. E 98, 032209 (2018).

[42] J. J. Pulikkottil, A. Lakshminarayan, S. C. L. Srivastava, A. Bäcker, and S. Tomsovic, Entanglement production by interaction quenches of quantum chaotic subsystems, Phys. Rev. E 101, 032212 (2020).

[43] F. L. Moore, J. C. Robinson, C. F. Bharucha, B. Sundaram, and M. G. Raizen, Atom Optics Realization of the Quantum $\delta$-Kicked Rotor, Phys. Rev. Lett. 75, 4598 (1995).

[44] D. L. Shepelyansky, Coherent Propagation of two Interacting Particles in a Random Potential, Phys. Rev. Lett. 73, 2607 (1994).

[45] K. Frahm, Eigenfunction structure and scaling of two interacting particles in the one-dimensional Anderson model, Eur. Phys. J. B 89, 115 (2016).

[46] M. O. Sales, W. S. Dias, A. Ranciaro Neto, M. L. Lyra, and F. A. B. F. de Moura, Sub-diffusive spreading and anomalous localization in a 2D Anderson model with off-diagonal nonlinearity, Solid State Commun. 270, 6 (2018).

[47] S. Nag, A. Lahiri, and G. Ghosh, Entropy production due to coupling to a heat bath in the kicked rotor problem, Phys. Lett. A 292, 43 (2001).

[48] E. Ott, T. M. Antonsen, and J. D. Hanson, Effect of Noise on Time-Dependent Quantum Chaos, Phys. Rev. Lett. 53, 2187 (1984).

[49] W. H. Zurek, Decoherence, einselection, and the quantum origins of the classical, Rev. Mod. Phys. 75, 715 (2003).

[50] D. H. White, S. K. Ruddell, and M. D. Hoogerland, Phase noise in the delta kicked rotor: From quantum to classical, New J. Phys. 16, 113039 (2014).

[51] S. Paul, S. Sarkar, C. Vishwakarma, J. Mangaonkar, M. S. Santhanam, and U. Rapol, Nonmonotonic diffusion rates in an atom-optics Lévy kicked rotor, Phys. Rev. E 100, 060201(R) (2019). 